LA EMPRESA FAMILIAR, EL CASO DE UNA EMPRESA RESTAURANTERA

\title{
LA EMPRESA FAMILIAR, EL CASO DE UNA EMPRESA RESTAURANTERA
}

FAMILY BUSINESS, THE CASE OF A RESTAURANT COMPANY

Laura Mariana Cázares Jiménez* Norma Aguilar Morales**

*Estudiante de Licenciatura en Administración. Universidad Juárez Autónoma de Tabasco.

**Doctora en Gestión Estratégica y Políticas de Desarrollo. Profesora investigadora de Universidad Juárez Autónoma de Tabasco. Email: gialca@hotmail.com

Dirección para recibir correspondencia: Imariana.cazares@gmail.com

Fecha de recibido: 20 de enero de 2018

Fecha de aceptación: 20 de marzo de 2018

CÁZARES-JIMÉNEZ L. M., AGUILAR-MORALES N.

ENERO-ABRIL 2018. Año 24, Número 68. Págs. 11-28 


\section{RESUMEN}

OBJETIVO: Identificar las creencias potencializadoras y limitantes que se tienen sobre la administración de un negocio familiar desde la mirada del dueño(a) para reflexionar sobre aquellas oportunidades o dificultades en los procesos administrativos que pudieran no ser conscientes en pro del crecimiento de la propia empresa.

MATERIAL Y MÉTODO: La investigación es un estudio de caso con investigación de campo, es de corte cualitativo, por medio de entrevista a profundidad, el instrumento empleado es el cuestionario de (Aguilar, Sandoval, Surdez, \& Gómez, 2013).

RESULTADOS: Se encontró que el dueño de la empresa tiene procedimientos administrativos escasos, su estructura administrativa es muy sencilla, no tiene un organigrama donde se pueda ver el nivel de jerarquía que manejan, hasta ahora no quiere nombrar a un sucesor. Se propone que la empresa cuente con una planeación estratégica, que le permita diferenciarse de la competencia, y así sirviéndole a su vez de ventaja competitiva, así como de soporte para su continuidad, se recomienda formalizar la estructura y funciones de la empresa, que permita una transición exitosa.

CONCLUSIONES: Con base a la información recabada se puede concluir que la empresa familiar carece de una formación empresarial, la cual le impide tener una concepción de las funciones administrativas que garantizan un funcionamiento eficiente del negocio; Sin embargo ha podido ocupar un lugar importante en el mercado regional y gracias a eso han podido ir creciendo, no cuenta con un registro de marca; la estructura de la empresa esta desequilibrada ya que no existe tal estructura y cada miembro de la familia no tiene un lugar específico de desempeño.

PALABRAS CLAVE: Gerencia familiar. PyMEs. Proceso administrativo. Relación empresafamilia. 


\section{ABSTRACT}

OBJECTIVE: To identify the potentializing and limiting beliefs about the administration of a family business from the owner's point of view to reflect on those opportunities or difficulties in the administrative processes that may not be considered to favor the company's growth.

MATERIAL AND METHOD: The research is a case study with field research, it is qualitative, through an in-depth interview, the instrument used is the questionnaire (Aguilar, Sandoval, Surdez, \& Gómez, 2013).

RESULTS: It was found that the owner of the company has very limited administrative procedures, his administrative structure is very simple, he does not have an organization chart where the level of hierarchy that they manage can be seen, so far, they do not want to name a successor. It is proposed that the company has a strategic planning, which allows the company to differentiate itself from the competition, and this planning will also work as a competitive advantage, as well as support for its continuity, it is recommended to formalize the structure and functions of the company, allowing a successful transition.

CONCLUSIONS: Based on the collected information, it can be concluded that the family business lacks a business training, which prevents it from having a conception of the administrative functions that guarantee an efficient operation of the business; however, this company has been able to occupy an important position in the regional market and thanks to that, it has grown, it does not have a trademark registration; the structure of the company is unbalanced, since there is no such structure no member of the family has a specific role to perform.

KEYWORDS: Family management. SMEs. Administrative process. Family-business relationship. 


\section{LA EMPRESA FAMILIAR, EL CASO DE UNA EMPRESA RESTAURANTERA}

\section{MARCO TEÓRICO}

Las PyMEs familiares pueden ser particulares, ya que cada una de ellas cuenta con sus propios valores, reglas y procedimientos.

En la literatura consultada, se puede observar que Goyzueta (2013) menciona que "Las empresas familiares predominan en muchos países a nivel mundial, especialmente en Latinoamérica. Están ligadas a nuestra vida económica y social, a tal punto que casi ya no nos damos cuenta de su presencia"; por eso se dice que, en múltiples países, las empresas familiares constituyen un elemento crucial dentro de su economía, ya que estas tienen una fundamental participación en la actividad empresarial, así como en la generación de empleo.

A su vez, Ramírez (2014) afirma que la empresa familiar es muy particular, anteriormente la empresa familiar se le veía como un sistema que integra dos subsistemas: la familia y la empresa. Cada subsistema cuenta con sus propios valores, reglas, políticas y procedimientos, y los problemas se dan cuando ambos subsistemas se cruzan en las actividades de una persona.

Por otra parte, las empresas familiares representan un importante apoyo para la economía en México, muchas de ellas insertas en las micros y medianas empresas. De acuerdo con el reporte de la Family Business (Magazine, 2014), diez de las 250 empresas familiares más grandes del mundo son mexicanas.

En México, las PyMEs han ayudado a las grandes ciudades y a que los pequeños poblados se urbanicen, a si logrando exportar en algunos casos sus productos, actualmente la mayoría de estas empresas son de carácter familiar debido a los cambios económicos que se viven.

En este sentido, (Cruz Corona, Paz Gomez, \& Camacho Gomez, 2016) mencionan que:

En México, como en muchos otros países, son numerosos los negocios familiares; es decir, las unidades de producción de bienes o prestación de servicios operadas por los miembros de una familia y las personas asimiladas a la familia y que dependen de los ingresos que se generan en el taller familiar.

A pesar del avance experimentado en las últimas décadas en el fenómeno de las PyMEs familiares, gran parte del camino está aún por recorrer, ya que no se cuenta con muchas investigaciones en la actualidad, y para lograr grandes adelantos en este campo la 
LA EMPRESA FAMILIAR, EL CASO DE UNA EMPRESA RESTAURANTERA

investigación deberá afrontar ciertas barreras teóricas y metodológicas. Entre ellas están, por ejemplo, la comprensión y la definición del propio concepto de PyME familiar.

\section{PyMEs familiares}

PyMe es el acrónimo de pequeña y mediana empresa, se trata de una empresa de cualquier giro (mercantil, industrial, entre otros) que tiene un número reducido de trabajadores y que registra ingresos moderados; este tipo de empresas fueron creadas a partir de la segunda guerra mundial debido a que la economía quedó muy afectada.

Una empresa familiar es un conjunto de actividades económicas, establecidos en un área geográfica específica, real o virtualmente- donde los miembros de la familia aportan recursos financieros, conocimientos y mano de obra para la creación de un bien o un servicio, por lo que se establece quien o quienes desarrollarán cada una de las actividades: tomar decisiones, cumplir tareas específicas, así como la forma de la distribución de las utilidades, que se requieren para el logro del éxito (Ramirez Martinez \& Lamoyi Bocanegra, 2015).

De igual manera, Guizar, (2009), citado por Longenecker (1994); señalan que una empresa familiar es aquella que reúne dos elementos:

1) En la parte de propiedad: todos o por lo menos la mayor parte de la propiedad, lo que permita mantener el control de la organización, deben pertenecer a dos o más miembros de una misma familia que normalmente son emprendedores natos.

2) En la que al menos dos de los miembros de la familia están involucrados en el funcionamiento de la empresa.

\section{Teorías y modelos de empresas familiares}

El modelo de los 3 círculos.

El modelo de los tres círculos fue desarrollado en un artículo seminal por John Davis y Renato Tagiuri en 1982 y menciona Arenas Cardona \& Rico Balvin (2014) que es un clásico que ha sido y sigue siendo extensamente utilizado y citado para explicar qué es y cómo funciona la empresa familiar. Sin embargo, probablemente poca gente lo ha leído en su versión original. 


\section{LA EMPRESA FAMILIAR, EL CASO DE UNA EMPRESA RESTAURANTERA}

Debido a esto (Perez Molina, 2012) menciona que:

Este modelo explica la forma en que se sobreponen los sistemas de la Familia, la Empresa y la Propiedad, así como el lugar que ocupan cada uno de los miembros que pertenece a alguno de los siete sub-conjuntos que se forman en las áreas de sus intersecciones.

En resumen, este modelo busca explicar la interacción que se presenta en una empresa familiar ilustrando la relación entre tres círculos de influencia: propiedad, familia y negocio, tal y como se observa en la siguiente imagen.

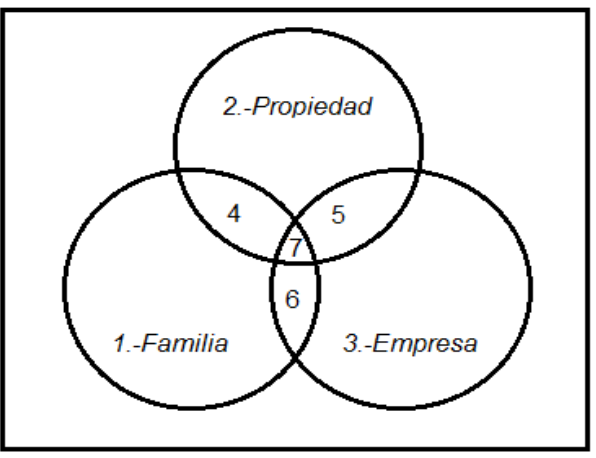

Figura 1. Modelo de los tres círculos.

Fuente: Elaboración propia con base en información obtenida de Davis y Tagiuri (1982).

Arenas et al., (2014) menciona que el modelo de los tres círculos da a conocer que la empresa familiar está regida por la interacción entre 3 círculos de influencia: familia, propiedad y empresa. El primero se refiere a lazos sanguíneos; el segundo, a accionistas, y el tercero, a funciones desempeñada dentro de la empresa. La interacción de dichas áreas, la falta de fronteras divisorias o el desconocimiento de las mismas provocan que haya conflictos aparentemente imposibles de resolver.

Cada una de las distintas áreas en el modelo de los 3 círculos representa diferentes agentes implicados en la empresa familiar. A continuación, y con base en (Goyzueta, 2013), se presenta de cada uno de ellos.

1. Miembros de la familia, que no son propietarios y no trabajan en la empresa;

2. Propietarios de la empresa, que no son miembros de la familia y no trabajan en la empresa; 
LA EMPRESA FAMILIAR, EL CASO DE UNA EMPRESA RESTAURANTERA

3. Empleados de la empresa, que no son miembros de la familia y no son propietarios;

4. Miembros de la familia, que son propietarios y no trabajan en la empresa;

5. Miembros de la familia, que no son propietarios y trabajan en la empresa;

6. Propietarios, que no son miembros de la familia y trabajan en la empresa; y

7. Miembros de la familia, que son propietarios y trabajan en la empresa.

Debido a esto Perez Molina (2012) dice que las principales ventajas de este modelo son que, además de resultar fácil de comprender, tiene una gran aplicación práctica. Ante cualquier situación conflictiva en una empresa familiar, resulta muy útil tomarse la molestia de dibujas estos tres círculos y ubicar a los individuos implicados en el sector que corresponda, ya que en esta propuesta todas y cada una de las personas que de alguna forma u otra tienen relación con una empresa familiar, pueden ubicarse en alguno de estos círculos y subconjuntos.

\section{El modelo de los cinco círculos.}

El modelo de los cinco círculos, es creado por el Profesor y autor español Amat Joan (2000), es una ampliación del modelo de los tres círculos, ya que se agrega dos aspectos más:

- La gestión de la empresa: hace referencia a la dimensión organizativa, a las perspectivas estratégicas y a la competitividad de la empresa en cuanto a productos, mercados y recursos.

- La sucesión que afecta directamente a las otras cuatro áreas.

A continuación, (Perez Molina, 2012) describe las cinco esferas básicas del modelo:

La Familia: Considera factores como los valores, actitudes, relaciones internas, pautas de comunicación, grado de armonía existente y su influencia en la empresa, grado de compromiso de la familia con la continuidad de la empresa, traslado de los temas familiares a la esfera de los negocios y viceversa.

La Propiedad: Estructura accionaria, grado de armonía entre los accionistas, gestión jurídica (civil, comercial y fiscal) del patrimonio, eficacia de los órganos de gobierno (asamblea de accionistas, junta directiva).

La Empresa: Considera la visión estratégica y la competitividad de la empresa familiar. 


\section{LA EMPRESA FAMILIAR, EL CASO DE UNA EMPRESA RESTAURANTERA}

La Gerencia: Considera los aspectos relacionados con la administración de los recursos humanos, tecnológicos y materiales.

La Sucesión: Es uno de los procesos más críticos que debe emprender toda empresa familiar para garantizar su continuidad en manos de la familia empresaria.

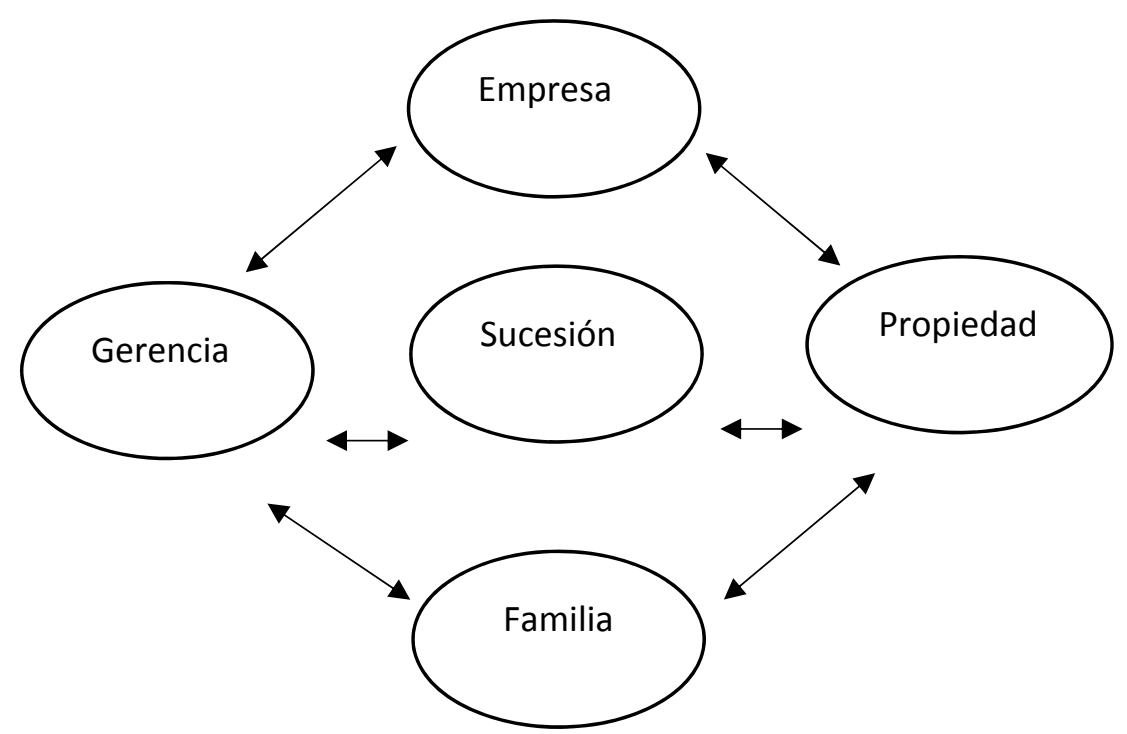

\section{Figura 2. Modelo de los cinco círculos.}

Fuente: Elaboración propia con base en información obtenida de Amat Joan (2000).

\section{Modelo de la triple hélice.}

El estudio entre Estado, Universidad y Empresa es analizado como un modelo propuesto por Etzkowitz y Leydesdorff (1997).

De acuerdo a ( Chang Castillo, 2010) mencionan que:

Este modelo pretende que el accionar de la Universidad sea un creador de conocimiento, que juega un papel primordial entre la relación empresa y gobierno; y como éstos se desarrollan para crear innovación en las organizaciones como fuente de creación del conocimiento. Este modelo es un proceso intelectual orientado a visualizar la evolución de las relaciones entre universidad, sociedad, y por otro lado caracterizado por la intervención de la universidad en los procesos económicos y sociales. 


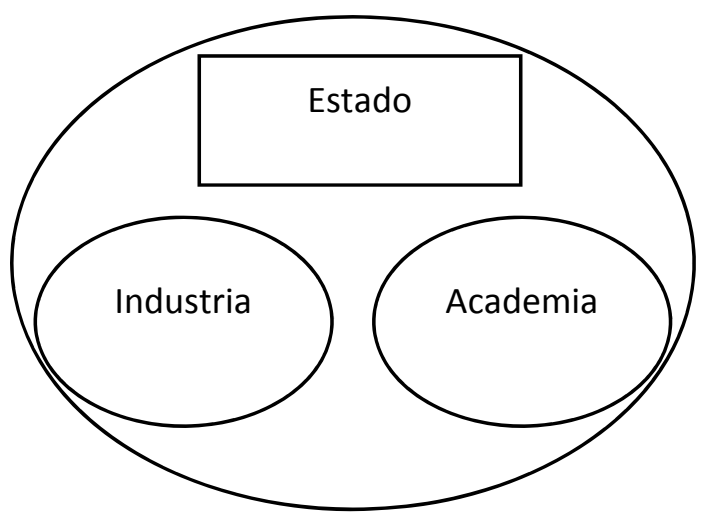

Figura 3. Modelo de la triple hélice.

Fuente: Elaboración propia con base en información obtenida de Etzkowitz, H. \&. Leydesforff, L. (2000).

\section{PLANTEAMIENTO DEL PROBLEMA}

Las empresas familiares han formado parte fundamental de la economía tanto en México como en el mundo. De acuerdo con Beltrán (2004; citado en Cruz, Mendoza y Pérez, 2015), "Las empresas familiares son de suma importancia dentro del mercado mundial, debido a que su participación se encuentra entre el 65\% y el 99\% en los países industrializados y del tercer mundo." Esto revalida que las PyMEs familiares son una gran fuente de crecimiento económico y social a nivel mundial, así como también menciona Tapies (2011) que "la empresa familiar es la forma de propiedad más extendida en el tejido empresarial internacional" (p.15).

Por otra parte, menciona Lazcano (2016) que "La influencia de la familia en Latinoamérica es muy fuerte" con esto se debe tener presente que en México la importancia de las PyMEs familiares va de acuerdo a la tendencia mundial, debido a que Serna (2012) alude que "las empresas de tamaño micro y pequeñas son la forma más extendida de unidades económicas en México y tienen en común que son empresas familiares" (p. 51). Todo este tipo de microempresas aporta diversas clases de actividades económicas (primarias, secundarias y terciarias); así de esta manera demostrando un gran desarrollo en nuestro país.

En este sentido, Cruz, et al. (2015) afirman que en una encuesta desarrollada por Confecamaras y la Superintendencia de Sociedades (2009) a empresas medianas y pequeñas, en la cual participaron 7,414 compañías de forma voluntaria, arrojó que el 81\% no poseen procedimientos documentados que permitan realizar una sucesión adecuada. Adicionalmente, indica que la mayoría de las microempresas familiares se encuentran a cargo de la primera 
LA EMPRESA FAMILIAR, EL CASO DE UNA EMPRESA RESTAURANTERA

generación con un $75.8 \%$, el $21.3 \%$ han logrado pasar a la segunda generación y el 2.9\% llegaron a la tercera (p. 16).

Por lo mencionado anteriormente, se pueden identificar que las PyMEs familiares tienen una mayor dificultad, para mantenerse por la falta de procesos a nivel administrativo, debido a que Belausteguigoitia (2003); citado en Priego Padrón, Paz Gomez, Camacho Gomez, y Méndez Olán, (2013) indica que los fenómenos en torno a la empresa familiar son muy variados según el lugar en donde desarrolle sus actividades. En México, las empresas familiares ocupan entre el 70 y $95 \%$ del total de las organizaciones, la tasa de mortandad de las Pymes ha sido del $80 \%$ durante los primeros dos años (p. 69).

Debido a los problemas que se presentan en este tipo de PyMEs, diversas investigaciones han demostrado que una de las dificultades que mayormente se muestran para su desarrollo es la falta de conocimiento o la mala aplicación que tienen en sus prácticas administrativas.

Las PyMEs normalmente carecen de una estructura formal en todas sus áreas, de acuerdo con Belausteguigoitia (2004; citado en Priego, et al. 2013), "estas empresas presentan problemas en su organización, financiamiento y desarrollo debido al escaso estudio de sus dirigentes" ( $p$. $68)$.

Gersick, Davis, Hampton y Lansberg (2006); citado en Meira y Carvahal, (2013) dicen que el nacimiento de las empresas familiares se da a partir de una idea proveniente de uno o varios miembros de la familia con visión emprendedora, y estas presentan dos motivaciones de los fundadores que tienen un impacto duradero sobre sus empresas, que son el deseo de ser propietario y no empleado, motivados por la independencia personal y el hecho de tener el control de la propia vida; y el deseo de encontrar la oportunidad y aprovecharla.

Una de las dificultades para definir lo que es una empresa familiar está relacionada con la complejidad de la superposición de los factores empresarial y familiar en la organización.

Por otra parte, Ramírez (2014) menciona que la empresa familiar, es una fusión de dos sistemas o dos instituciones, el familiar y el empresarial. El sistema familiar es profundamente emocional, mientras que el empresarial es de base laboral. En las empresas familiares, estos dos sistemas se superponen y llegan a ser interdependientes, lo cual causa dificultades por ser dos sistemas opuestos, con objetivos y prioridades diferentes. 
LA EMPRESA FAMILIAR, EL CASO DE UNA EMPRESA RESTAURANTERA

Debido a lo expuesto anteriormente, se busca que una vez identificadas las necesidades de las empresas, sus gerentes o ya sean las personas encargadas directamente, tengan claras las alternativas administrativas que se están implementando actualmente en las microempresas, con el fin de lograr realizar grandes cambios, que permita a sus compañías ser en realidad competitivas.

Al estudiar parte de los aspectos que intervienen en los procesos administrativos, se hace necesario tener estrategias claras, que permitan a los microempresarios su ejecución, sin que estas sean vistas como un imposible, para que puedan tener una mejora continua, con los recursos que la microempresa cuente.

De igual manera Hurtarte (1998); citado en Ramírez (2014) hace mención que cuando se piensa en empresas familiares, se entiende a creer que la empresa fue fundada por un empresario con una visión específica, que se esfuerza por sacarla adelante y sueña con heredarla a sus hijos. Cuando los hijos se involucran en aquello que el padre formó con tanto esfuerzo, luchan por hacerlo más grande y productivo, llevándolo muchas veces al punto máximo del negocio. Sin embargo, existe una tendencia que indica que al llegar la tercera generación esta etapa de crecimiento se termina y la empresa familiar tiende a disminuir su crecimiento por la diversidad de opiniones e intereses de los familiares involucrados.

Se llega a pensar que esto lleva a muchas empresas familiares, que en su primera y segunda generación fueron exitosas, a disminuir su éxito en el mercado y muchas veces, a la quiebra.

Las microempresas familiares por su naturaleza están enfrentando distintos obstáculos para poder trasladar la dirección de la empresa de una generación a otra. Hoy en día, los potenciales sucesores tienen más oportunidades de estudio y autorrealización fuera de la empresa. Esto crea una fuerte competencia para la empresa, que debe ser más atractiva sobre las otras opciones, de lo contrario el fracaso en la sucesión del poder se empeora. Por consiguiente, el cierre de la empresa se vuelve inmediato u obliga a esta a realizar un cambio drástico que hace perder a la empresa su característica familiar.

Por ello, es importante crear estrategias toda vez que se hayan analizado los resultados obtenidos en la encuesta que se aplicó, para que los microempresarios puedan acceder a asesorías profesionales sin incurrir en altos costos y tener un desarrollo en su microempresa, ya 
LA EMPRESA FAMILIAR, EL CASO DE UNA EMPRESA RESTAURANTERA

que se debe tener en cuenta que muchos microempresarios inician su empresa de forma informal y no profesional.

A través de la siguiente investigación surge la siguiente pregunta:

¿Cuáles son las creencias que se tienen sobre administrar una empresa familiar?

\section{OBJETIVO DE INVESTIGACIÓN}

\section{GENERAL}

Identificar las creencias potencializadoras y limitantes que se tienen sobre la administración de un negocio familiar, desde la mirada del dueño(a) para reflexionar sobre aquellas oportunidades o dificultades en los procesos administrativos que pudieran no ser conscientes en pro del crecimiento de la propia empresa.

\section{ESPECÍFICO}

- Conocer los antecedentes de las PyMEs familiares de Villahermosa, Tabasco.

- Conocer cómo se maneja la jerarquía en las PyMEs familiares.

- Conocer cómo se maneja la toma de decisiones en las PyMEs familiares.

- Conocer que procedimientos administrativos usan las PyMEs familiares.

\section{MATERIAL Y MÉTODO}

\section{DISEÑO DE INVESTIGACIÓN}

La investigación es un estudio de caso con investigación de campo, es de corte cualitativo, por medio de entrevista a profundidad, el instrumento empleado es el cuestionario de Aguilar, Sandoval y Surdez (2014). La técnica de recolección de la información empleada fue la entrevista semi-estructurada.

\section{POBLACIÓN Y MUESTRA}

En el presente trabajo, el sujeto en el estudio es el propietario de la empresa, a quien se entrevistó. 


\section{INSTRUMENTO DE MEDICIÓN}

Se empleó el cuestionario de Aguilar, Sandoval y Surdez (2014). La entrevista se divide en seis etapas de la cuales habla de las siguientes variables:

1. Datos sociodemográficos.

2. Datos de la empresa.

3. Dedicación a la empresa.

4. La empresa y la familia.

5. Actitudes emprendedoras.

\section{RESULTADO}

La actividad que desarrolla la empresa, es un restaurante que se dedica a la creación de platillos con pescados y mariscos, se entrevistó al propietario.

Una vez realizada la entrevista y transcrita la grabación del audio, se procedió a analizar la información y se obtuvieron las categorías plasmadas en la figura número 4.

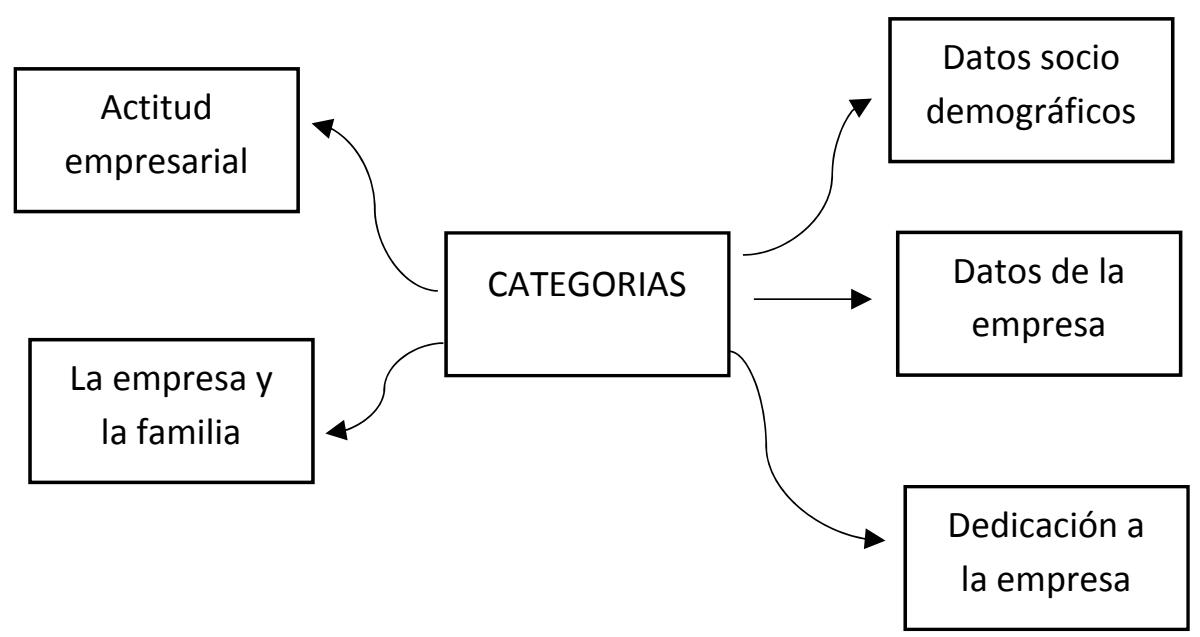

Figura 4. Categorías de análisis de la entrevista.

Fuente: Elaboración propia con base de Aguilar, Sandoval y Surdez (2014). 
LA EMPRESA FAMILIAR, EL CASO DE UNA EMPRESA RESTAURANTERA

La definición de cada categoría es la siguiente:

- Datos sociodemográficos: son datos personales del propietario.

- Datos de la empresa: son datos directamente relacionados con la constitución y operación de la empresa.

- Dedicación a la empresa: tiempo que el propietario dedica a la administración de su negocio.

- La empresa y la familia: es la forma en que el propietario distribuye tanto tiempo y recursos entre empresa y la familia.

- Actitud empresarial: tiene que ver con aquellos atributos personales que lo identifican como emprendedor y como empresario.

En la figura 5, se pueden observar los componentes que integran cada categoría y que fueron producto del análisis de la entrevista. 


Capacidad de resolución de
problemas.
Conocimientos requeridos:
Planeación estratégica a corto
plazo.
El inició la empresa.
Le gusta el riesgo ya que dice
que en las decisiones siempre
hay un riesgo y en ese riesgo
hay una posibilidad de crecer.
Acepta sugerencias.
No tiene total autonomía en la
toma de decisiones, lo
comparte con la familia.
Lleva una agenda de sus
compromisos.
No interviene directamente en
el proceso productivo.
Resiste largas jornadas de
trabajo.
Buena relación con el
personal valora el recurso
humano.
Para él es muy importante el
éxito económico, pero también
en reconocimiento social.
No tiene un organigrama por
su familia.

No tiene antecedentes familiares empresariales. Participación de gran parte de la familia en el negocio, como apoyo moral y administración de la empresa

Reinvierte el capital, gran parte de las utilidades se utiliza en el negocio para mejorar el establecimiento e ir creciendo y otra parte en la familia.

No ha contemplado la

sucesión, y aún no tiene un sucesor.

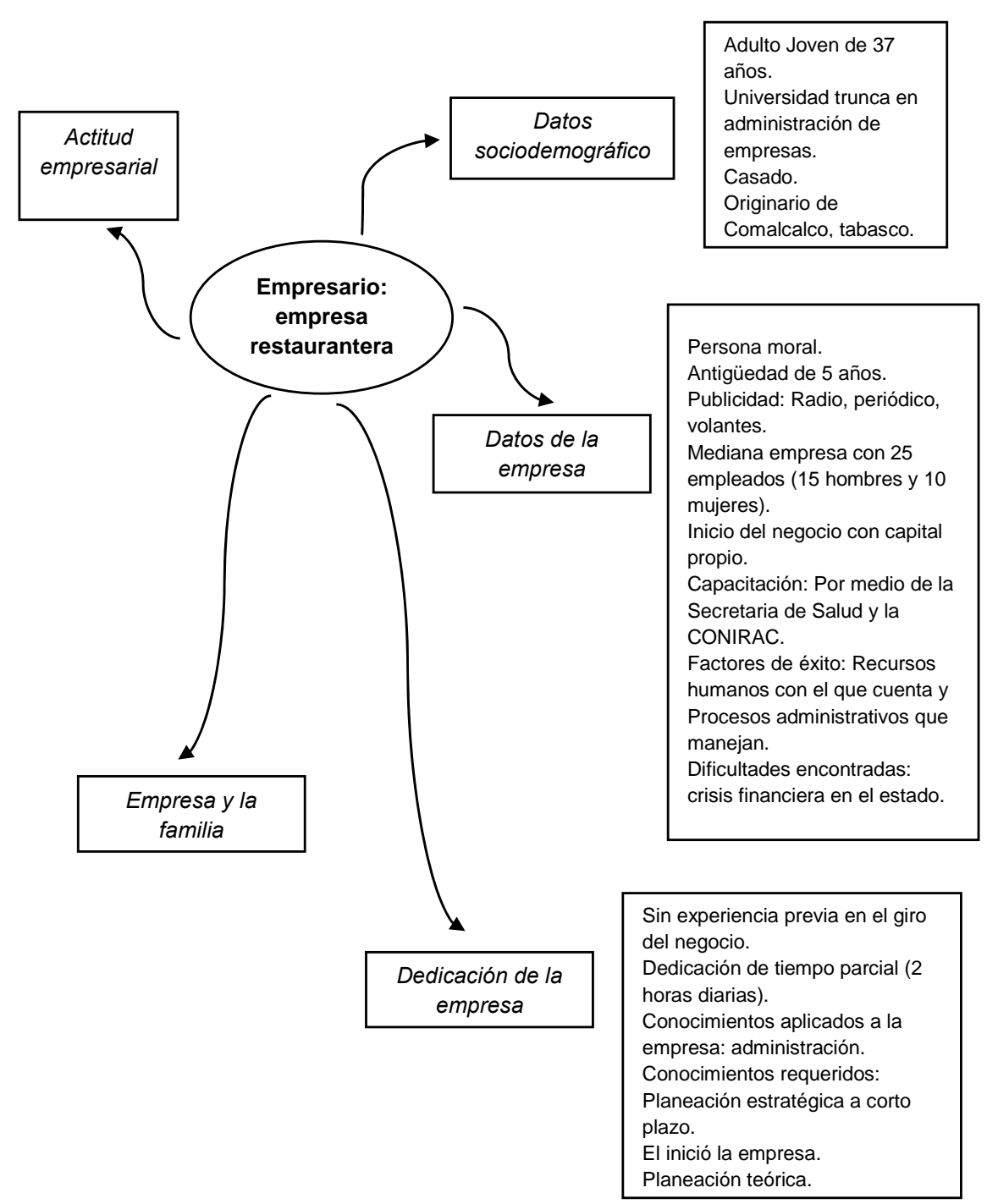

Figura 5. Análisis de la entrevista a la empresa restaurantera de mariscos.

Fuente: Elaboración propia.

En la figura 5, se puede observar el análisis de la entrevista realizada al empresario, quien es propietario de un restaurante de Mariscos. Cabe mencionar, que es un adulto joven, posee un estudio de universidad trunca en administración de empresas, su empresa cuenta con 5 años en el mercado y maneja una variedad de productos en su menú, cuenta con 25 empleados (15 hombres y 10 mujeres), tiene constantemente rotación de personal, pero a pesar de no contar con una formación universitaria completa ha sabido administrar bien su negocio, se asigna un sueldo fijo para cubrir sus necesidades personales y las de su familia, lo que ha permitido 
LA EMPRESA FAMILIAR, EL CASO DE UNA EMPRESA RESTAURANTERA

reinvertir sus ganancias para hacer crecer la empresa. En la toma de decisiones siempre trata de involucrar a su familia quien lo ha apoyado tanto moralmente como en la administración de la empresa, ya que algunos colaboran con él en el negocio.

\section{CONCLUSIONES}

Al analizar los resultados obtenidos en la entrevista, se pudo constatar lo mencionado en López (2003), dice que:

La empresa familiar es un dominio netamente familiar, en el que suele dominar una lógica económica diferente de la que determina los procesos en una empresa capitalista, ya que antes de perseguir la obtención de una ganancia, la empresa familiar tiene como objetivo prioritario garantizar la subsistencia de una familia. Este rasgo distintivo marca desde el inicio las actividades de la empresa familiar, condicionando sus posibilidades de continuidad y eventual expansión.

Debido a esto, la continuidad y los procesos de la empresa familiar, son más complejos que los de las empresas que no tienen una organización de tipo familiar, ya que de ello depende el tiempo de vida de las mismas, el funcionamiento y crecimiento a través del tiempo.

Se puede decir, que esta PyME familiar restaurantera carece de una formación empresarial, ya que al ser una empresa de primera generación, esto le impide tener una idea de las funciones administrativas que les garantice un funcionamiento eficiente de la empresa y tome en cuenta la importancia de una estructura organizativa, y que garantice su continuidad generacional. El microempresario prácticamente realiza las actividades de manera empírica.

Finalmente, el estilo de gestión del empresario presenta ciertas carencias, sobre todo de conocimientos, ya que no concede mucha importancia a un organigrama y esto llega a ocasionar conflictos entre la familia, además no quieren invertirle tiempo o no lo quieren hacer por miedo a problemas en cuestiones jerárquicas. Sin embargo, a pesar de la falta de conocimientos profesionales ha sabido llevar al éxito su negocio y lograr la permanencia en un giro en donde no contaba con experiencia previa. 


\section{REFERENCIAS BIBLIOGRÁFICAS}

Aguilar, N., Sandoval, M., Surdez, E., \& Gómez, S. (2013). Characteristics or entrepreneurs of small business in Tabasco, Mexico. Review of Business \& Finaance Studies, 4(1), pp. 23-36.

Amat, J. (2000). Modelo de los cinco círculos. La continuidad de la empresa familiar. 2. Barcelona: Gestión 2000.

Arenas Cardona, H. A., \& Rico Balvin, D. (2014). La empresa familiar, el protocolo y la sucesión familiar. Estudios Gerenciales, 30(132), pp. 252-258. Obtenido de file:///F:/9\%20SEMESTRE/SEMINARIO/ARTICULOS/ART\%20MOD\%203\%20CIRCULO S.htm

Chang Castillo, H. G. (2010). El modelo de la triple hélice como un medio para la vinculación entre la universidad y empresa. Nacional de Administración, 1(1),pp. 85-94. Obtenido de https://pdfs.semanticscholar.org/8cdc/70d3edd4b603b53229eb09f4c37e7ce82d2a.pdf

Cruz Corona, L. L., Paz Gomez, C. A., \& Camacho Gomez, M. (2016). PLAN DE SUCESIÓN EN EMPRESAS FAMILIARES. EL CASO DE UNA PANIFICADORA. HITOS(63), 114139. Obtenido de http://revistas.ujat.mx/index.php/hitos/article/view/1845/1497

Cruz, Z., Mendoza, N. y Pérez, A. (2015). Diagnóstico de los procesos administrativos de las pequeñas empresas familiares manufactureras, en la localidad de barrios unidos de la ciudad de Bogotá d.c; Tesis para obtener el grado de Licenciatura de Administración de empresas, Facultad de Ciencias Administrativas y Contables, Universidad de la Salle, Bogotá. Recuperado de http://repository.lasalle.edu.co/bitstream/handle/10185/17114/11102037_2015.pdf?sequ ence $=1$

Goyzueta, S. I. (2013). Modelo de gestión para las empresas familiares con perspectivas de crecimiento y sostenibilidad. Perspectivas, 16(31), pp. 87-132. Obtenido de http://www.scielo.org.bo/scielo.php?script=sci_arttext\&pid=S1994-37332013000100003

Guizard Montufar, R. (2008). ¿Empresas familiares emprendedoras en México? Ide@s CONCYTEG, 3(33), pp. 3-13. Obtenido de http://www.concyteg.gob.mx/ideasConcyteg/Archivos/33022008_EMPRESAS_FAMILIA RES_EMPRENDEDORAS_MEXICO.pdf

Hurtarte, M. E. (1998). Empresa familiar: La nueva perspectiva de un sistema de gobernanza integral de familiar, propiedad y empresa y su aplicación en Guatemala. Tesis para obtener el grado de Licenciatura en administración, Facultad de Ciencias administrativas, Universidad Francisco Marroquín. Guatemala 
LA EMPRESA FAMILIAR, EL CASO DE UNA EMPRESA RESTAURANTERA

Lazcano Alvarez, K. M. (2016). afectaciones de la cultura sobre la toma de decisiones del microempresario familiar. ResearchGate, pp. 1-23. Obtenido de https://www.researchgate.net/publication/311645764_AFECTACIONES_DE_LA_CULTU RA_SOBRE_LA_TOMA_DE_DECISIONES_DEL_MICROEMPRESARIO_FAMILIAR

Lopez, G. A. (2003). Estudio de la empresa familiar en Rosario. Una aproximación etnográfica. INVENIO, pp. 91-100. Obtenido de file:///C:/Users/Erick/Downloads/DialnetEstudioDeLaEmpresaFamiliarEnRosario-3330716.pdf

Ramírez, V. (2014). Proceso de sucesión en empresas familiares, caso: Empresas de purificadoras de agua. Tesis para obtener grado de Licenciatura de Administración de empresas, Facultad de ciencias económicas y empresariales, Universidad Rafael Landívar, Guatemala. Recuperado de http://biblio3.url.edu.gt/Tesario/2014/01/01/Ramirez-Lesly.pdf

Meira Teixeira, R., \& Carvalhal, F. (2013). Sucesión y conflictos en empresas familiares. Estudios y perspectivas en turismo, 22(5), pp. 854-874. Obtenido de http://www.redalyc.org/pdf/1807/180728713003.pdf

Pérez Molina, A. I. (2012). El modelo de empresa familiar: Los cuatro pilares fundamentales. Investigación y pensamiento critico, 1(3), pp. 2-12. Obtenido de https://dialnet.unirioja.es/servlet/articulo?codigo $=4817932$

Priego Padrón, J., Paz Gomez, C. A., Camacho Gomez, M., \& Méndez Olán, L. A. (2013). Algunas consideraciones sobre la administración de las pequeñas empresas panaderas familiares en la ciudad de Villahermosa, Tabasco. HITOS, 19(54), pp. 67-76. Obtenido de http://revistas.ujat.mx/index.php/hitos/article/view/111

Ramírez Martinez, M. A., \& Lamoyi Bocanegra, C. L. (2015). La sucesión en las empresas familiares: Sector de agua purificada. PRZEDSIĘBIORCZOŚĆ I ZARZĄDZANIE, 16(7), pp. 81-94. Obtenido de http://piz.san.edu.pl/docs/e-XVI-7-1.pdf

Serna, M. G. (2012). Empresas familiares frente a las crisis. Economia, sociedad y territorio, 7(38), pp. 43-80. Obtenido de http://www.redalyc.org/articulo.oa?id=11122403003

Tapies, J. (2011). Empresa familiar: Un enfoque multidisciplnar. Universia business review(32), pp. 12-25. Obtenido de http://www.redalyc.org/articulo.oa?id=43320760001 